\title{
Hot-Electron Transport, Noise, and Power Dissipation in GaN Channels at High Density of Electrons
}

\author{
A. Matulionis ${ }^{a}$, J. Liberis $^{a}$, M. Ramonas ${ }^{a, b}$ \\ AND I. MATUliONIENE $\dot{\mathrm{E}}^{a}$ \\ ${ }^{a}$ Fluctuation Research Laboratory, Semiconductor Physics Institute \\ A. Goštanto 11, Vilnius 01108, Lithuania \\ ${ }^{b}$ EIT4, Bundeswehr University, 85577 Neubiberg, Germany \\ The experimental results on transport, noise, and dissipation of electric \\ power for voltage-biased Si-doped GaN channels are compared with those of \\ Monte Carlo simulation. The measured dissipated power shows a stronger \\ hot-phonon effect than the simulated one. On the other hand, the exper- \\ imental results on the electron drift velocity at high electric fields show a \\ weaker hot-phonon effect as compared with the simulated one. The misfit \\ can be reduced if a conversion of the friction-active nonequilibrium longitu- \\ dinal optical phonons into the friction-passive longitudinal optical phonons \\ is considered.
}

PACS numbers: 72.20.Ht, 72.70.+m, 72.80.Ey

\section{Introduction}

Emission of longitudinal optical (LO) phonons by hot electrons is the main power dissipation mechanism in GaN at high electric fields. As a result, a large part of the supplied electric power is transferred to the LO-phonon subsystem, and the latter is displaced from equilibrium. "Hot phonons" is a short term for this situation. The accumulated hot phonons cause different effects: introduce additional friction, reduce the electron drift velocity, slow down electron energy dissipation [1]. The hot-phonon effects manifest themselves at a high density of electrons in channels subjected to high electric fields [2]. The hot-phonon problem is of great interest for microwave high-power field-effect transistors.

Measuring microwave noise of hot electrons is a convenient way for experimental investigation of hot-phonon effects in voltage-biased channels [3]. Complicated disintegration of hot phonons into other vibration modes is usually in- 
terpreted in terms of a single parameter - the lifetime. The noise technique has provided with the hot-phonon lifetime [3] in an excellent agreement with the value obtained from time-resolved experiment on the intersubband absorption assisted by LO phonons [4]. For GaN and GaN-based channels, the lifetime is measured at different electron temperatures $[3,5]$, lattice temperatures $[5,6]$, and electron densities [7]. Our goal is to illustrate, through comparison of the experimental results [8] with those of Monte Carlo simulation [2], that the single-lifetime approach fails, and the experimental investigation of noise, transport, and power dissipation can provide with more features of the hot-phonon disintegration in GaN.

\section{Results and discussion}

Stars in Fig. 1 illustrate the electron velocity estimated from the data on current, electron density, and channel dimensions (stars [8]) measured at room temperature for Si-doped GaN channels. The velocity approaches the value of $3 \times 10^{7} \mathrm{~cm} / \mathrm{s}$ at electric field $300 \mathrm{kV} / \mathrm{cm}$. According to the simulation [2], hot phonons reduce the velocity (Fig. 1, solid curve). A longer hot-phonon lifetime causes a lower drift velocity (bullets). At fields above $50 \mathrm{kV} / \mathrm{cm}$, solid line is below the experimental data (stars) - the simulated hot-phonon effect is stronger than the experiment shows.

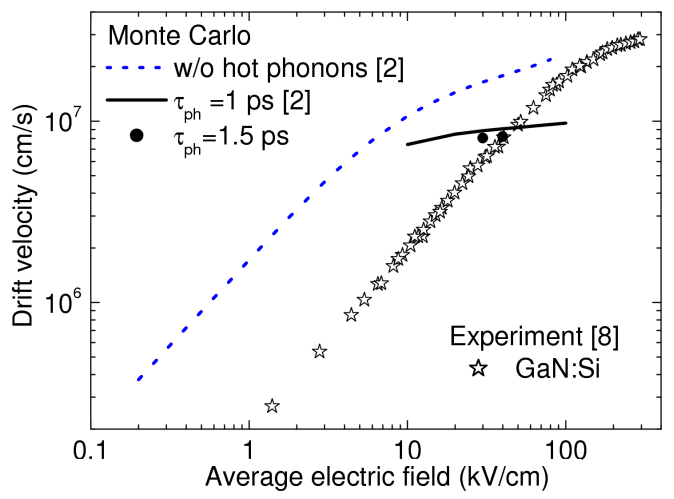

Fig. 1. Drift velocity against electric field for GaN at electron density of $10^{18} \mathrm{~cm}^{-3}$ : experiment (stars [8]) and Monte Carlo simulation (lines [2], and bullets).

The simulated dissipated power per electron depends on the mean kinetic energy of the electrons (curves, Fig. 2). The experimental data (stars [8]) obey a similar nearly-exponential dependence on the inverse noise temperature. The activation energy is close to the LO-phonon energy.

The simulation (Fig. 2, solid line) shows a weaker hot-phonon effect than the experiment (stars). This contradicts with the conclusion obtained from the analysis of the electron drift velocity (Fig. 1). 


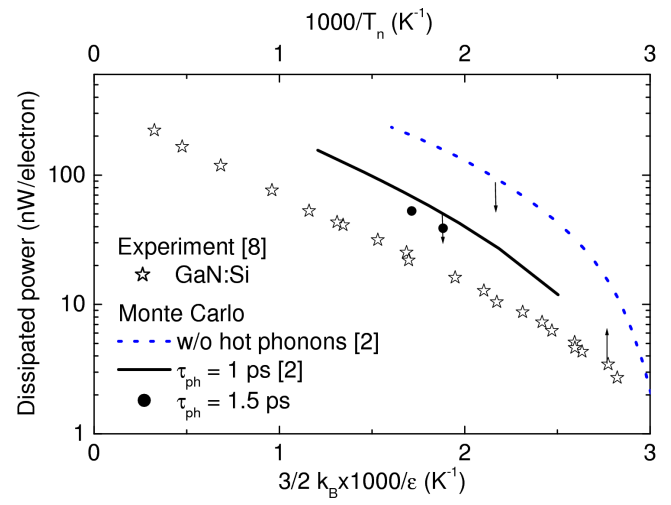

Fig. 2. Dissipated power per electron against reciprocal noise temperature for Si-doped GaN (stars [8]) and reciprocal mean kinetic energy (lines [2], bullets).

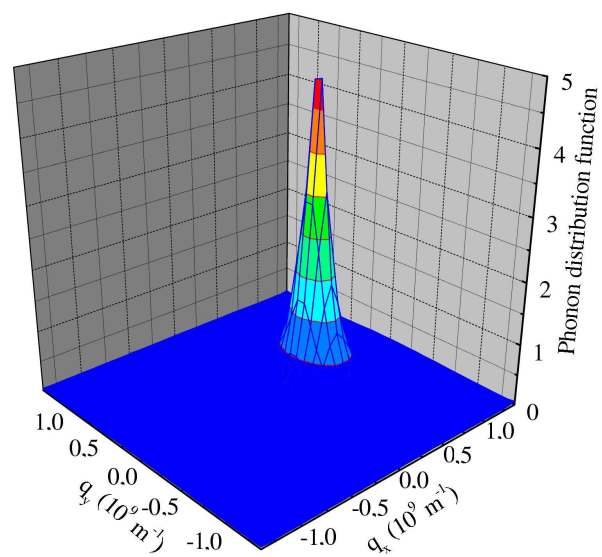

Fig. 3. Hot-phonon distribution function at $100 \mathrm{kV} / \mathrm{cm}$ for $\mathrm{GaN}$ at electron density of $10^{17} \mathrm{~cm}^{-3}$.

The contradiction is reduced if LO-phonon-LO-phonon collisions (LO-LO scattering) are taken into account. The hot-phonon effect on electron drift velocity (the additional friction) results from the nonequilibrium occupancy of the LO-phonon modes allowed by energy and momentum conservation (Fig. 3). Let us call them the friction-active LO-phonon modes. The high occupancy of the friction-active modes causes the strong hot-phonon effect on the electron drift velocity. On the other hand, in the hot-phonon lifetime approach, the hot- phonon disintegration rate is independent of the shape of their distribution if the total hot-phonon number remains unchanged. Supposing that the hot-phonon distribution were wider (washed out by the possible LO-LO scattering neglected during the simulation), the occupancy of the friction-active modes would reduce. This would reduce the friction, and a better agreement with the experimental data on drift velocity would be obtained. 
In conclusion, the experimental data on transport, noise and dissipation contain information on the rate of conversion of the friction-active LO-phonon modes into the friction-passive LO-phonon modes.

\section{References}

[1] A. Matulionis, Phys. Status Solidi A 203, 2313 (2006).

[2] M. Ramonas, A. Matulionis, L.F. Eastman, Semicond. Sci. Technol. 22, 875 (2007).

[3] A. Matulionis, J. Liberis, I. Matulionienè, M. Ramonas, L.F. Eastman, J.R. Shealy, V. Tilak, A. Vertiatchikh, Phys. Rev. B 68, 035338 (2003).

[4] Z. Wang, K. Reimann, M. Woerner, T. Elsaesser, D. Hofstetter, J. Hwang, W.J. Schaff, L.F. Eastman, Phys. Rev. Lett. 94, 037403 (2005).

[5] A. Matulionis, J. Liberis, L. Ardaravičius, L.F. Eastman, J.R. Shealy, A. Vertiatchikh, Semicond. Sci. Technol. 19, S421 (2004).

[6] K.T. Tsen, D.K. Ferry, A. Botchkarev, B. Sverdlov, A. Salvador, H. Morkoç, Appl. Phys. Lett. 72, 2132 (1998).

[7] K.T. Tsen, J.G. Kiang, D.K. Ferry, H. Morkoç, Appl. Phys. Lett. 89, 112111 (2006).

[8] J. Liberis, M. Ramonas, O. Kiprijanovič, A. Matulionis, N. Goel, J. Simon, K. Wang, H. Xing, D. Jena, Appl. Phys. Lett. 89, 202117 (2006). 